\title{
Covid-19: Cases of inflammatory syndrome in children surge after urgent alert
}

\author{
Elisabeth Mahase
}

The BMJ

There has been a surge in cases of an inflammatory syndrome in children, believed to be linked to covid-19, following an alert to doctors in the UK at the end of April. ${ }^{1}$

The syndrome, which in the UK is referred to as the "paediatric inflammatory multisystem syndrome which is temporally associated with SARS-CoV-2," has also been seen in Italy and the US and has been compared with both Kawasaki disease and toxic shock.

Experts have said that the condition may be an "antibody mediated or delayed response" to covid-19 that happens several weeks after the infection. They also stressed, however, that while doctors must know what to look out for, the syndrome is rare and has good outcomes.

Kawasaki disease is a rare condition which mainly affects children under five and is characterised by a high temperature that lasts for five days or more, a rash, and swollen glands in the neck. It can cause the blood vessels to become inflamed and swollen, and can lead to complications in the coronary arteries.

\section{Thirty fold increase}

In the province of Bergamo, Italy, researchers have reported a 30 fold increased incidence of Kawasaki like disease since the start of the covid-19 outbreak.

In a study published in the Lancet $^{2}$ they said that between 18 February and 20 April this year, 10 children, with an average age of 7.5, were diagnosed with the syndrome compared with 19 children, with an average age of three, who were diagnosed in the five years leading up to the pandemic (1 January 2015 to 17 February 2020).

Among the post-covid-19 group, eight of the 10 children were positive for SARS-CoV-2 antibodies- $\operatorname{IgG}$ or IgM, or both.

The team reported that the rate of new cases was 0.3 per month in the pre-pandemic group, and 10 per month in the post-pandemic group. Among the covid-19 group more children had cardiac symptoms (6 out of 10), Kawasaki disease shock syndrome (5 out of 10), macrophage activation syndrome (5 out of 10), and the need for adjunctive steroid treatment (8out of 10). In the pre-covid-19 group only two of 19 children had cardiac involvement and just three required adjunctive steroid treatment.

\section{A distinct syndrome}

Julia Kenny, a consultant in paediatric infectious diseases and immunology at Evelina London Children's Hospital, said that the Italian findings appear consistent with cases seen in the south east of England.

"Evelina has more than 50 children with a similar syndrome and while very few tested positive for the virus on swabs, the majority tested positive for covid-19 antibodies when subsequently tested, suggesting their disease is associated with exposure to the virus," she said.

At Great Ormond Street Hospital (GOSH), clinicians said they have seen a surge in cases following the alert but stressed that numbers were still small and that parents shouldn't panic.

Paediatric infectious diseases consultant Karyn Moshal said, "There are a number of these children coming through to the district general hospitals and we're getting calls to discuss them. Although some are well enough to be managed where they are, we are erring on the side of caution and bringing them in, so we can treat them early."

She said many of the children fall "between toxic shock and Kawasaki disease in terms of their presentation," but that it does seem to be a distinct syndrome.

Moshal added, "The age group is a much older than the classic Kawasaki patient group, who would be under five with half of them under two. The population we are seeing are seven or eight through to 17, with the majority in their teens, around 13 or 14. The abdominal pain, the diarrhoea, and the gastrointestinal symptoms together with a persistent fever are particularly prominent in this group."

Sophie Skellett, paediatric and neonatal intensive care consultant at GOSH, said that her department was seeing more patients with toxic shock type symptoms, but with "some sort of heart involvement." She said that many patients were "presenting with suspected appendicitis."

\section{Delayed response to SARS-CoV-2}

Speaking at a press briefing on the syndrome on 13 May, Liz Whittaker, clinical lecturer in paediatric infectious diseases and immunology at Imperial College London, highlighted that the peak in these cases appears to be several weeks behind the peak of covid-19 across the country. 
"In London, the peak was estimated around the first or second week of April, and we think we saw the peak of these children this or last week. What we wonder is whether this is an antibody mediated or a delayed response to the virus that is happening several weeks after the infection. That would explain why these children don't test positive for the virus using polymerase chain reaction tests," she said.

Whittaker said that while the syndrome is referred to as "temporarily associated with SARS-CoV-2" and that they cannot definitely say it is covid-19 related, "it is happening in the middle of a pandemic so it seems reasonable to suggest they are related."
Correction: The syndrome is referred to as the paediatric inflammatory multisystem syndrome which is temporally [not "temporarily"] associated with SARS-CoV-2. We corrected this article on 27 May 2020.

1 Mahase E. Covid-19: concerns grow over inflammatory syndrome emerging in children. BMJ 2020;369:m1710. 10.1136/bmj.m1710 32345602

2 Verdoni L, Mazza A, Gervasoni A, et al. An outbreak of severe Kawasaki-like disease at the Italian epicentre of the SARS-CoV-2 epidemic: an observational cohort study. 13 May 2020, Lancet. www.thelancet.com/journals/lancet/article/PIIS0140-6736(20)31103-X/ fulltext.

This article is made freely available for use in accordance with BMJ's website terms and conditions for the duration of the covid-19 pandemic or until otherwise determined by BMJ. You may use, download and print the article for any lawful, non-commercial purpose (including text and data mining) provided that all copyright notices and trade marks are retained.

https://bmj.com/coronavirus/usage 